\title{
Third dimension of a map
}

\author{
Karel Jedlička*
}

Department of Geomatics, University of West Bohemia, Pilsen, Czechia, smrcek@kgm.zcu.cz

Plan4all.eu, Czechia karel.jedlicka@ plan4all.eu

Wirelessinfo living lab, Litovel, Czechia, jedlicka@wirelessinfo.cz

* Corresponding author

Keywords: Thematic maps, Atlas, Statistical Abstract

\begin{abstract}
:
A communication of information from its producer to a user can be done in many ways which differs in their effectivity. Although a map is a native communication channel for presenting of spatial data, a presentation of spatial data in $3 \mathrm{D}$ environment is a growing alternative in recent years. Therefore this chapter describes basic principles of dealing with third dimension in a map.
\end{abstract}

Note that this contribution focuses on matters related purely to GIS. The contribution is not going to describe advanced $3 D$ scene visualization techniques such as shadow modelling, ray-tracing, etc. (as they belong to computer graphics).

Also a type of display in not addressed in detail. Just to mention in general, having a 3D scene, it can be displayed on:

1. Monitor - then it is perspective visualization. A perception of depth emerges by moving the virtual observer position or line of sight.

2. Monitor using passive or active glasses / 3D monitor - a full stereo perception emerges.

3. 3D print / hologram - physical/virtual three-dimensional scene emerges.

Coming to GIS related issues. First of all, there exists solutions displaying $\mathbf{2 . 5}$ dimensional data and they are commonly called 3D GIS as well even if they do not support full 3D objects.

Next, there are two different types of coordinate systems used: a Cartesian coordinate system, speaking geographically - a projected coordinate system, e.g. an UTM Zone. Then all coordinates are in the same units (usually meters). More challenging is the other type - a geographic coordinate system using latitude and longitude locating both objects and phenomena on Earth globe, but metric units for (ellipsoidal or orthometric) height - then different types of units (angular and metrical) are used for different axes. Such a situation is typical for virtual globes.

Talking about cartography, there are two main approaches to visualize a 3D scene, photorealistic versus symbolized). Photorealistic approach tends to use real world textures and create an impression as close to real world as possible. The other approach, consisting of applying a cartographic symbols on the scene, challenges contemporary cartography, as not all cartographic techniques can be transferred from 2D to 3D. There can be distinguished among techniques able to apply without a change, techniques need to be adapted and inapplicable techniques.

And last but not least major issue is that 3D GIS has to deal with large data in a different way than in 2D. Two dimensional GIS deals with large amount of data by a concept of scale dependent rendering (also called a scale dependent map) and using pyramiding and generalization techniques. But important thing is that always the whole thematic layer (or even a map) is represented at one level of detail in one view. When zoom in or out, an appropriate level of pyramid is depicted. A different approach is needed in 3D - instead of using one zoom level as a threshold for what and how to visualize, the observer position and line of sight have to be taken into account. Then, objects closer to observer have to be presented in more detailed way than objects away, no matter if they are from the same layer or different layers.

The research reported in this paper has been supported by following project:

- Sustainability support of the centre NTIS - New Technologies for the Information Society, LO1506, Czech Ministry of Education, Youth and Sports 